\title{
A Liaison between Sudden Sensorineural Hearing Loss and SARS-CoV-2 Infection
}

\author{
Job Harenberg ${ }^{1,2}$ Jost B. Jonas ${ }^{1}$ Eleonora M. C. Trecca ${ }^{3}$
}

\author{
${ }^{1}$ Medical Faculty Mannheim, Ruprecht Karls University, Heidelberg, \\ Germany \\ 2 DOASENSE GmbH, Heidelberg, Germany \\ ${ }^{3}$ Department of Otolaryngology-Head and Neck Surgery, \\ University Hospital of Foggia, Foggia, Italy \\ Thromb Haemost 2020;120:1237-1239.
}

\section{Microthrombosis and Sudden Sensorineural Hearing Loss}

Microthrombosis is one of the multifactorial processes involved in the development of vascular, malignant, and neuroinflammatory diseases and organ failure. It is caused by endothelial cell dysfunction, which is induced by local coagulation disorders followed by platelet dysfunction and fibrin formation, and septicemia followed by local hypoxemia and cytokine activation. ${ }^{1}$ The main triggers of microthrombosis include the following.

- Acquired coagulation disorders and inherited thrombophilia.

- Platelet, leucocyte, or erythrocyte dysfunctions.

- Endothelial dysfunctions and local endovascular hyperviscosity (-Fig. 1). ${ }^{2}$

Microvascular dysfunction plays an important role in the pathogenesis of hearing disorders, such as sudden sensorineural hearing loss (SSNHL), in which hearing is lost within 72 hours. It affects 5 to 27 persons per 100,000 people every year. The main audiometric criterion currently used to diagnose SSNHL is a reduction in hearing of $\geq 30 \mathrm{~dB}$ in three contiguous audiometric frequencies. When premorbid audiometry is not available, the hearing threshold is usually compared with that of the contralateral ear. Up to $90 \%$ of SSNHL cases are idiopathic, and the most accredited etiologies are viral, vascular, and autoimmune. ${ }^{3}$

\section{Serotonin as a Driver of Sudden Sensorineural Hearing Loss}

In this issue, Drouet et al validated plasma serotonin in SSNHL. ${ }^{4}$ The authors showed that an increased plasma serotonin concentration could distinguish between a discovery SSNHL group, a validation SSNHL group, and a control

Address for correspondence Job Harenberg, MD, DOASENSE GmbH, Waldhofer Strasse 102, D-69123 Heidelberg, Germany (e-mail: j.harenberg@doasense.de).

group, with $96 \%$ specificity and $90 \%$ sensitivity. The authors suggested that an increased plasma serotonin concentration may be associated with the development of SSNHL.

This study reminds us that low to moderate titers of antiphospholipid antibodies and high levels of factor VIII/von Willebrand factor are involved in the pathophysiology of SSNHL. Thrombophilia caused by inherited mutations, such as the G20210A prothrombin mutation, G1619A factor V Leiden mutation, and C677T methyltetrahydrofolic acid transferase mutation, may not be involved in the development of SSNHL. The authors also showed that atherosclerosis may be involved in SSNHL as indicated by elevated levels of homocysteine, but this association was statistically weak.

The authors retrospectively compared a discovery group of 133 SSNHL patients with a validation group of 128 newly diagnosed SSNHL patients and an age- and sex-matched control group. Unlike in the SSNHL groups, systemic disorders such as arterial hypertension, diabetes mellitus, hyperlipidemia, smoking, and family history of cardiovascular disease were prevalent in the control group. Another limitation of the study was the rapid development and disappearance of SSNHL symptoms. Furthermore, the prevalence of deafness differed between the discovery group (40\%) and the validation group (76\%). As SSNHL may have occurred before the patients first noticed the symptoms and because plasma serotonin concentrations were assessed after serotonin- and tryptophan-rich foods were excluded for 48 hours, the presented results may not represent the true serotonin concentration at the time of SSNHL onset.

\section{Multifactorial Etiology of Sudden Sensorineural Hearing Loss}

Other pathophysiological investigations have identified factors that may contribute to SSNHL development, in addition to those described by Drouet et al. ${ }^{4}$

(c) 2020 Georg Thieme Verlag KG Stuttgart . New York
DOI https://doi.org/ 10.1055/s-0040-1714370. ISSN 0340-6245. 
- T allele carriers of platelet glycoprotein Ia (GPIa) C807T polymorphism (associated with myocardial infarction, stroke, and retinal vein thrombosis) and elevated fibrinogen levels were reported as risk factors for SSNHL. ${ }^{5}$

- TheT-786C, G894T, and 4a/4b polymorphisms in the endothelial nitric oxide synthetase genes were more prevalent in SSNHL patients, and these may induce plasma hyperviscosity and increased erythrocyte deformability. ${ }^{6}$

- Whole-blood viscosity and red blood cell aggregation were increased in patients with SSNHL. ${ }^{7}$

- Plasminogen activator inhibitor -1 levels and anticardiolipin antibodies were elevated in patients with SSNHL. ${ }^{8}$

- Interestingly, the laboratory atherosclerosis risk factors (cholesterol, triglycerides, and homocysteine) were elevated in patients with SSNHL. ${ }^{8,9}$

\section{SARS-CoV-2 Infection and Sudden Sensorineural Hearing Loss}

After the manuscript had been accepted for publication, the severe acute respiratory syndrome coronavirus 2 (SARS-CoV2) outbreak started, ${ }^{10}$ and data became available on the coincidence of SSNHL and SARS-CoV-2 infection. The latter has been associated with various thrombosis-related complications, both venous and arterial, leading to a systemic multiorgan condition. ${ }^{11,12}$ The associated viral sepsis affects many organs via angiotensin-converting enzyme 2 (ACE2) receptors and ultimately results in multiple fatal organ failures, ${ }^{13}$ which may contribute to the pathophysiology of SSNHL.

SSNHL is caused by viral infection, vascular occlusion, abnormal cellular stress responses within the cochlea, and immune-mediated mechanisms. ${ }^{14}$ SARS-CoV-2 enters the cell via ACE2 receptor, ${ }^{15}$ and can infect many organs, including the central nervous system, peripheral nervous system, and hearing center in the temporal lobe. ${ }^{16}$ The virus promotes excess cytokine release and induces hearing damage, ${ }^{17}$ endotheliitis, and systemic impaired microcirculatory function. ${ }^{18}$ The virus also invades the cochlear nerve, causing neuritis, and soft tissues of the cochlea, causing cochleitis. ${ }^{19}$ SARS-CoV-2 infection has now been identified in patients with SSNHL who have clinically relevant reductions in highfrequency pure-tone thresholds and transiently evoked otoacoustic emission amplitudes (- Fig. 1)..$^{13,20-22}$

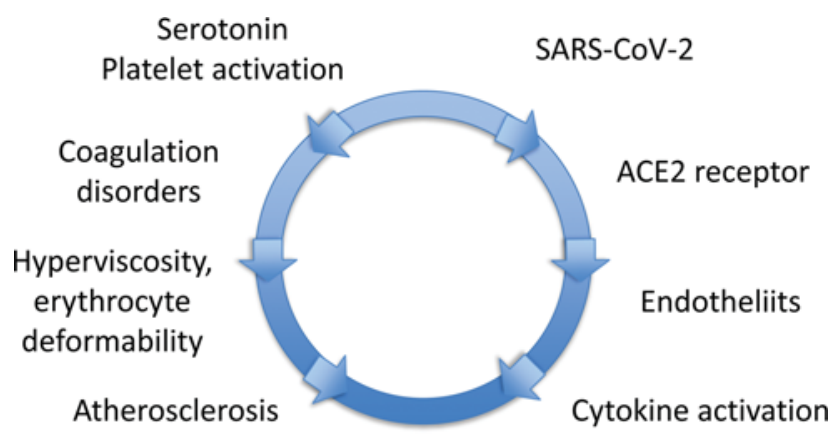

Microthrombosis

Fig. 1 Interplay of serotonin, blood coagulation, and SARS-CoV-2 infection on sudden sensorineural hearing loss.

\section{What the Future May Offer}

Serotonin is a sensitive and specific marker for SSNHL diagnosis, and probably activates platelets in the microcirculation. SARS-CoV-2 infection appears to cause endotheliitis in the hearing center of the temporal lobe, the cochlear nerve, and tissues of the cochlea. Serotonin release and SARS-CoV-2 infection intertwine to activate platelets and drive SSNHL, and this needs to be tackled by specific interventions.

\section{Conflict of Interest \\ None declared.}

\section{References}

1 Evans CE. Hypoxia and HIF activation as a possible link between sepsis and thrombosis. Thromb J 2019;17:16

2 Mangin PH, Gardiner EE, Nesbitt WS, et al; Subcommittee on Biorheology. In vitro flow based systems to study platelet function and thrombus formation: recommendations for standardization: communication from the SSC on biorheology of the ISTH.J Thromb Haemost 2020;18(03):748-752

3 Chandrasekhar SS, Tsai Do BS, Schwartz SR, et al. Clinical practice guideline: sudden hearing loss (update) executive summary. Otolaryngol Head Neck Surg 2019;161(02):195-210

4 Drouet L, Hautefort $\mathrm{CH}$, Vitaux $\mathrm{H}$, et al. Plasma serotonin is elevated in adult patients with sudden sensorineural hearing loss. Thromb Haemost 2020;120(09):1291-1299

5 Rudack C, Langer C, Stoll W, Rust S, Walter M. Vascular risk factors in sudden hearing loss. Thromb Haemost 2006;95(03): 454-461

6 Mannini L, Cecchi E, Fatini C, et al. Clinical haemorheology and microcirculation. Ann Ist Super Sanita 2007;43(02): 144-155

7 Becatti M, Marcucci R, Mannucci A, et al. Erythrocyte membrane fluidity alterations in sudden sensorineural hearing loss patients: the role of oxidative stress. Thromb Haemost 2017;117(12): 2334-2345

8 Marcucci R, Alessandrello Liotta A, Cellai AP, et al. Cardiovascular and thrombophilic risk factors for idiopathic sudden sensorineural hearing loss. J Thromb Haemost 2005;3(05):929-934

9 Yang Z, Shi J, He Z, et al. Predictors for imaging progression on chest CT from coronavirus disease 2019 (COVID-19) patients. Aging (Albany NY) 2020;12(07):6037-6048

10 Harenberg J, Favaloro E. COVID-19: progression of disease and intravascular coagulation - present status and future perspectives. Clin Chem Lab Med 2020;58(07):1029-1036

11 Zhai Z, Li C, Chen Y, et al; Prevention Treatment of VTE Associated with COVID-19 Infection Consensus Statement Group. Prevention and treatment of venous thromboembolism associated with coronavirus disease 2019 infection: a consensus statement before guidelines. Thromb Haemost 2020;120(06):937-948

12 Spiezia L, Boscolo A, Poletto F, et al. COVID-19-related severe hypercoagulability in patients admitted to intensive care unit for acute respiratory failure. Thromb Haemost 2020;120(06):998-1000

13 Bikdeli B, Madhavan MV, Gupta A, et al; Global COVID-19 Thrombosis Collaborative Group. Pharmacological agents targeting thromboinflammation in COVID-19: Review and implications for future research. Thromb Haemost 2020:120(07):1004-1024

14 Trecca EMC, Gelardi M, Cassano M. COVID-19 and hearing difficulties. Am J Otolaryngol 2020;41(04):102496

15 Violi F, Pastori D, Cangemi R, Pignatelli P, Loffredo L. Hypercoagulation and antithrombotic treatment in coronavirus 2019: a new challenge. Thromb Haemost 2020;120(06):949-956 
16 Mao L, Jin H, Wang M, et al. Neurologic manifestations of hospitalized patients with coronavirus disease 2019 in Wuhan, China. JAMA Neurol 2020;77(06):1-9

17 Cure E, Cumhur Cure M. Comment on "Hearing loss and COVID19: a note”. Am J Otolaryngol 2020;41(04):102513

18 Varga Z, Flammer AJ, Steiger P, et al. Endothelial cell infection and endotheliitis in COVID-19. Lancet 2020;395(10234):1417-1418

19 Chen X, Fu YY, Zhang TY. Role of viral infection in sudden hearing loss. J Int Med Res 2019;47(07):2865-2872
20 Mustafa MWM. Audiological profile of asymptomatic Covid-19 PCR-positive cases. Am J Otolaryngol 2020;41(03):102483

21 Sriwijitalai W, Wiwanitkit V. Hearing loss and COVID-19: a note. Am J Otolaryngol 2020;41(03):102473

22 Karimi-Galougahi M, Naeini AS, Raad N, Mikaniki N, Ghorbani J. Vertigo and hearing loss during the COVID-19 pandemic - is there an association? Acta Otorhinolaryngol Ital 2020. Doi: 10.14639/0392-100X-N0820 